\title{
A UNICITY THEOREM FOR MEROMORPHIC MAPPINGS BETWEEN ALGEBRAIC VARIETIES \\ BY
}

\author{
S. J. DROUILHET
}

\begin{abstract}
Using the techniques of value distribution theory in several complex variables, we obtain a theorem which can be used to determine whether two nondegenerate meromorphic mappings from an affine algebraic variety to a projective algebraic variety of the same or lower dimension are identical. The theorem generalizes a result of $\mathbf{R}$. Nevanlinna in one complex variable.
\end{abstract}

1. Introduction. In 1926, R. Nevanlinna [12] proved that if $f, g: \mathbf{C} \rightarrow \mathbf{P}^{1}(\mathbf{C})$ are nonconstant holomorphic maps such that, for five points $a_{1}, \ldots, a_{5} \in \mathbf{P}^{1}, f^{-1}\left(a_{i}\right)$ $=g^{-1}\left(a_{i}\right)$ as point sets, then $f$ and $g$ are identical. He investigated analogous problems, and H. Cartan obtained related results [2], [3], [4], [13]. E. M. Schmid [14] studied questions of this type for holomorphic maps from open to compact Riemann surfaces. H. Fujimoto [6]-[9], has recently obtained generalizations of certain theorems of this type to the case of several complex variables. J. Carlson [1] has also studied unicity questions. In this paper we give another extension of Nevanlinna's theorem to the several variables setting. Our method consists in applying the value distribution theory results of Shiffman [15] to the idea underlying the original proof of Nevanlinna's theorem. Our main result follows.

THEOREM 1.1. Let $M$ be a smooth $n$-dimensional affine algebraic variety and $V$ be a smooth $k$-dimensional projective algebraic variety with $k \leqslant n$. Suppose $f, g: M \rightarrow V$ are nondegenerate meromorphic maps. Let $i: V \rightarrow \mathbf{P}^{N}(\mathbf{C})$ be a holomorphic map of $V$ into any projective space, so that $i \circ f$ and $i \circ g$ are meromorphic. Let $A$ be any hypersurface in $V$ with normal crossings such that the holomorphic line bundle $L_{A}$ on $V$ defined by $A$ is positive. If the following conditions are met, then $i \circ f \equiv i \circ g$ :

(1) Either $M=\mathbf{C}^{n}$ or both $f$ and $g$ are transcendental.

(2) As point sets $f^{-1}(A)=g^{-1}(A)$.

(3) For all $z$ in $f^{-1}(A)$ lying in the common domain of determinacy of $f$ and $g$, $f(z)=g(z)$.

(4) If $H$ denotes the hyperplane bundle on $\mathbf{P}^{N}$, then $L_{A} \otimes K_{V} \otimes\left(i^{*} H\right)^{-2}>0$.

The notation and terminology used in the statement of this theorem will be explained in the next section. Applications of this result to the specific cases where $V$ is $\mathbf{P}^{k}(\mathbf{C})$ or an elliptic curve will be given in $\$ 4$. $\$ 5$ will discuss the effects of weakening various hypotheses of the theorem.

Received by the editors February 2, 1979 and, in revised form, July 23, 1980.

AMS (MOS) subject classifications (1970). Primary 30A70, 32H25.

Key words and phrases. Value distribution theory, unicity theorem. 
This paper extends results in the author's doctoral thesis written at Rice University in 1974 under the direction of R. O. Wells, Jr. A weaker version of Theorem 1.1 was announced in [5]. The author would like to thank James Carlson and Wilhelm Stoll for helpful suggestions pertaining to the paper.

2. Résumé of background results. In this section we review and reproduce definitions, notation, and results from Shiffman [15] to be used in the proof of 1.1. For further details, the reader should consult that paper.

Definition 2.1. (a) If $z=\left(z_{1}, \ldots, z_{n}\right) \in \mathbf{C}$, then $|z|=\left(\left|z_{1}\right|^{2}+\ldots+\left|z_{n}\right|^{2}\right)^{1 / 2}$.

(b) $d^{c}=i(\bar{\partial}-\partial)$.

Definition 2.2. Let $V$ be a compact Kähler manifold.

(a) A $(1,1)$ form $\omega$ on $V$ is positive (semipositive) if locally $\omega$ can be expressed as $i / 2 \sum_{i, j} h_{i j} d z_{i} \wedge d \bar{z}_{j}$ where $\left(h_{i j}\right)$ is a positive definite (semidefinite) matrix.

(b) If $\alpha \in H^{2}(V, \mathbf{R})$, we say $\alpha>0(\geqslant 0)$ if $\alpha$ can be represented by a positive (semipositive) $(1,1)$ form.

(c) If $\alpha, \beta \in H^{2}(V, \mathbf{R})$ then

$$
[\alpha / \beta]=\inf \{k \in[-\infty, \infty]: k \beta-\alpha \geqslant 0\} .
$$

(d) A holomorphic line bundle $L$ on $V$ is positive if its first Chern class $c_{1}(L)$ satisfies $c_{1}(L)>0$.

(e) If $L$ is a holomorphic line bundle on $V$ with a hermitian metric $h$, then the curvature form $\eta$ of $h$ is given by $\eta=-(1 / 4 \pi) d d^{c} \log h$.

(f) $K_{V}$ denotes the canonical line bundle on $V$, and $K_{V}^{*}$ its dual.

Definition 2.3. In (e) if $h$ is any hermitian metric on $L$, then its curvature form represents $c_{1}(L)$. Conversely, any $(1,1)$ form representing $c_{1}(L)$ is the curvature form for some metric on $L$.

Definition 2.4. Let $X, Y$ be complex manifolds.

(a) $\mathscr{D}^{\prime p, q}(Y)$ denotes the space of currents of type $(p, q)$ on $Y$.

(b) If $A$ is a pure $(n-1)$ dimensional analytic subvariety of $Y$, then $[A] \in \mathscr{D}^{\prime 1,1}$ is the current given by integration over the regular points of $A$. In the interests of notational simplicity, we will sometimes denote $[A]$ by $A$ itself.

(c) A divisor on $Y$ is a current $D \in \mathscr{Q}^{\prime 1,1}(Y)$ of the form $D=\sum m_{j}\left[V_{j}\right]$ where the $m_{j}$ are integers and the $V_{j}$ are irreducible components of a complex hypersurface in $Y$. $D$ is positive if all $m_{j}>0$. If $D$ is positive or 0 we write $D>0$. If $D_{1}, D_{2}$ are divisors, we write $D_{1} \geqslant D_{2}$ if $D_{1}-D_{2} \geqslant 0$. If $D=\sum m_{j}\left[V_{j}\right]$ and the $V_{j}$ are distinct, $\operatorname{supp} D=\cup_{j} V_{j}$.

(d) If $f$ is a meromorphic function on $Y$, the divisor of $f$ is $\operatorname{Div} f=\sum a_{j}\left[V_{j}\right]-$ $\sum b_{j}\left[W_{j}\right]$ where $f$ has zeros of multiplicity $a_{j}$ on $V_{j}$ and poles of multiplicity $b_{j}$ on $W_{j}$.

(e) If $L$ is a holomorphic line on $Y, \Gamma(L)$ denotes the holomorphic sections of $L$. If $\sigma \in \Gamma(L)$, we may define the divisor Div $\sigma$ on $Y$ as locally being the divisor of any holomorphic function representing $\sigma$ with respect to a local trivialization of $L$. $|L|$ will denote the set of all divisors on $Y$ defined by elements of $\Gamma(L)$. If $D$ is a positive divisor on $Y$, then it defines a holomorphic line bundle on $Y$, denoted by $L_{D}$, such that $D \in\left|L_{D}\right|$. 
(f) Let $f: X \rightarrow Y$ be holomorphic, and $D$ be a divisor on $Y$ defined by a meromorphic function (or holomorphic section of a line bundle) $g$ on $Y$. Suppose $f(X) \nsubseteq D$. Then $f^{*} D$ is the divisor on $X$ defined as $f^{*} D=\operatorname{Div}(g \circ f)$.

(g) A holomorphic function $f: X \rightarrow Y$ is nondegenerate if $f$ has maximal rank at some point of $X$.

(h) If $\operatorname{dim} X=\operatorname{dim} Y$ and $f: X \rightarrow Y$ is a nondegenerate holomorphic map, then $R_{f}$, the ramification divisor of $f$, is the divisor on $X$ defined by the zeros of the Jacobian determinant of $f$ in local coordinates.

Definition 2.5. Let $X$ and $Y$ be complex manifolds with $Y$ compact.

(a) A meromorphic map $f: X \rightarrow Y$ is a holomorphic map $f_{0}: X_{0} \rightarrow Y$ defined on an open dense set $X_{0} \subset X$ such that the closure of the graph of $f_{0}$ in $X \times Y$ is an analytic subvariety of $X \times Y$. We can, and always will, assume that $X-X_{0}$ is an analytic set of codimension at least two in $X$.

(b) If $A \subset Y, f^{-1}(A)$ will denote $f_{0}^{-1}(A)$.

(c) If $g: X \rightarrow Y$ is a second meromorphic map given by a holomorphic $\tilde{g}_{0}$ : $\tilde{X}_{0} \rightarrow Y$ as in (a), then the common domain of determinacy of $f$ and $g$ is the set $E=X_{0} \cap \tilde{X}_{0}$. If $A \subset Y$, by abusing notation, we will write $f^{-1}(A)=g^{-1}(A)$ to mean $f^{-1}(A) \cap E=g^{-1}(A) \cap E$. By $f \equiv g$ we will mean $f(z)=g(z)$ for all $z$ in $E$.

(d) Continuing with the notation of (a), if $D$ is a divisor in $Y$, then $f^{*} D$ is the unique divisor on $X$ such that $\left.f^{*} D\right|_{X_{0}}=f_{0}^{*} D$.

(e) $f: X \rightarrow Y$ is nondegenerate if $f_{0}: X_{0} \rightarrow Y$ is.

(f) If $\operatorname{dim} X=\operatorname{dim} Y$ and $f$ is nondegenerate, $R_{f}$ is the unique divisor on $X$ such that $\left.R_{f}\right|_{X_{0}}=R_{f_{0}}$.

(g) If $\eta$ is a $C^{\infty}(1,1)$ form on $Y$, let $f_{0}^{*} \eta$ be the usual pullback on $X_{0}$. Let $f^{*} \eta$ be the extension of $f_{0}^{*} \eta$ to $X$ as an absolutely continuous current in $\mathscr{D}^{1,1}(X)$.

Definition 2.6. Let $Y$ be a complex manifold. An analytic hypersurface $A$ in $Y$ has normal crossings if $A$ is locally defined by an equation of the form $z_{1} \cdots z_{k}=$ 0 where $\left(z_{1}, \ldots, z_{n}\right)$ are local coordinates on $Y$.

DEFINITION 2.7. (a) Let $M$ be an $n$-dimensional smooth affine variety in $\mathbf{C}^{m}$. By making a suitable linear change of coordinates in $\mathbf{C}^{m}$, it can be assumed that the projection $\pi: M \rightarrow \mathbf{C}^{n}$ onto the first $n$ coordinates is proper. Choose a $C^{\infty}$ function $\sigma: \mathbf{C}^{n} \rightarrow \mathbf{R}$ such that for some $r_{0}>0, \sigma(z)=\log |z|$ for $|z|>r_{0}$ and $\sigma(z)<\log r_{0}$ if $|z|<r_{0}$. Let $\tau: M \rightarrow \mathbf{R}$ be $\tau=\sigma \circ \pi$, and let $B=\left\{x \in M: \tau(x)>\log r_{0}\right\}$. Then $\tau$ is plurisubharmonic on $B$, and the complement of $B$ is compact.

(b) Let $\alpha$ be the $(1,1)$ form on $M$ given by $\alpha=(1 / 2 \pi) d d^{c} \tau$. Set $\alpha^{n-1}=\alpha$ $\wedge \cdots \wedge \alpha[(n-1)$ times $]$.

(c) Suppose $\Phi \in \mathscr{D}^{1,1}(M)$ is such that $d \Phi=0$ and $\Phi$ is representable by integration. Let $\tau_{r}=\max (\log r-\tau, 0)$. Define $N(\Phi, r)=\left(\Phi, \tau_{r} \alpha^{n-1}\right)$, i.e. the real number obtained by applying $\Phi$ to $\tau_{r} \alpha^{n-1}$.

Definition 2.8. Let $M$ be a smooth affine algebraic variety as in 2.7 , and let $V$ be a smooth projective algebraic variety. Let $L$ be a holomorphic line bundle on $V$ equipped with some metric whose curvature form is $\eta$. Let $D \in|L|$. Suppose $f$ : $M \rightarrow V$ is meromorphic and $f(M) \notin D$. We define

(a) $T_{f}(L, r)=N\left(f^{*} \eta, r\right)$, 
(b) $N_{f}(D, r)=N\left(f^{*} D, r\right)$

for any real positive $r$.

If any other metric is used to define $\eta$, then $T_{f}(L, r)$ will change only by the addition of a bounded term. Thus, up to a bounded term, $T_{f}(L, r)$ depends only on $L$. Occasionally we will speak of $T_{f}(L, r)$ without specifying a metric on $L$.

The two principal results of Shiffman we will use are corollaries of his First and Second Main Theorems. They are found in [15] as Corollary 2.4, and Theorem 3.2 together with the first remark following it.

THEOREM 2.9. Let $M$ be a smooth affine algebraic variety as in 2.7. Suppose $V$ is $a$ smooth compact projective algebraic variety, $L$ is a hermitian holomorphic line bundle on $V$, and $D \in|L|$. Suppose $f: M \rightarrow V$ is meromorphic and $f(M) \nsubseteq \operatorname{supp} D$. Then

$$
N_{f}(D, r) \leqslant T_{f}(L, r)+O(1) .
$$

THEOREM 2.10. Let $M$ be a smooth $n$-dimensional affine algebraic variety as in 2.7, and let $V$ be a smooth $k$-dimensional projective algebraic variety with $k<n$. Let $A$ be an analytic hypersurface in $V$ with normal crossings. Suppose the holomorphic line bundle $L_{A}$ on $V$ defined by $A$ is positive. Let $f: M \rightarrow V$ be a nondegenerate meromorphic map. Let $\pi: M \rightarrow \mathbf{C}^{n}$ be the projection of 2.7. Let $\left(z_{1}, \ldots, z_{n}\right)$ be the usual coordinates on $\mathbf{C}^{n}$. Define $\hat{f}: M \rightarrow V \times \mathbf{C}^{n-k}$ by $\hat{f}=\left(f, z_{1} \circ \pi, \ldots, z_{n-k} \circ \pi\right)$. Suppose either $M=\mathbf{C}^{n}$ or $f$ is transcendental. Then

$$
\| T_{f}\left(L_{A}, r\right)-N_{f}(A, r)+T_{f}\left(K_{V}, r\right) \leqslant-N\left(R_{\hat{f}}, r\right)+o\left(T_{f}\left(L_{A}, r\right)\right) .
$$

Here $\|$ means that the in quality holds for all $r$ greater than some $r_{1}>0$, outside an open set $I \subset\left(r_{1}, \infty\right)$ such that $\int_{I} d x / x<\infty$.

THEOREM 2.11. From (3.1) in [15] it follows that, for the hypotheses in 2.10, $\lim _{r \rightarrow \infty} T_{f}\left(L_{A}, r\right)=\infty$.

In the proof of Theorem 4.2 we will also need the following application of Shiffman's defect relation, which follows immediately from the statement and proof of his Theorem 4.3 in [15].

THEOREM 2.12. If the conditions of 2.10 hold, then

$$
\liminf _{r \rightarrow \infty}\left[1-\frac{N_{f}(A, r)}{T_{f}\left(L_{A}, r\right)}\right] \leqslant\left[c_{1}\left(K_{V}^{*}\right) / c_{1}\left(L_{A}\right)\right] .
$$

The following fact is obtained by a straightforward calculation using the definitions (cf. Griffiths-King [10, Proposition 5.8]).

Proposition 2.13. Let $M$ be a smooth affine algebraic variety as in 2.7.

(a) Let $V_{1}, V_{2}$ be smooth projective algebraic varieties with holomorphic line bundles $L_{1}, L_{2}$ respectively. Let $\pi_{i}: V_{1} \times V_{2} \rightarrow V_{i}$ be projection on the ith factor. Suppose $f: M \rightarrow V_{1}$ and $g: M \rightarrow V_{2}$ are meromorphic. Then for $f \times g: M \rightarrow V_{1} \times$ $V_{2}$,

$$
T_{f \times g}\left(\pi_{1}^{*} L_{1} \otimes \pi_{2}^{*} L_{2}, r\right)=T_{f}\left(L_{1}, r\right)+T_{g}\left(L_{2}, r\right)+O(1)
$$


(b) If $V$ is a smooth algebraic variety and $f: M \rightarrow V$ is meromorphic, then for holomorphic line bundles $L_{1}, L_{2}$ on $V$,

$$
T_{f}\left(L_{1} \otimes L_{2}, r\right)=T_{f}\left(L_{1}, r\right)+T_{f}\left(L_{2}, r\right)+O(1)
$$

Here the results hold for any choice of hermitian metrics in the line bundles involved.

3. Proof of Theorem 1.1. In this section, we will use the results of Shiffman given in the previous section to prove Theorem 1.1. To prove our result, we will assume that all hypotheses of the theorem hold, but that $i \circ f$ and $i \circ g$ are not (essentially) the same function; a contradiction will follow.

Suppose that $f, g: M \rightarrow V$ are given by holomorphic maps $\tilde{f}_{0}: M_{0}^{\prime} \rightarrow V, \tilde{g}_{0}$ : $M_{0}^{\prime \prime} \rightarrow V$, where $M_{0}^{\prime}$ and $M_{0}^{\prime \prime}$ play the role of $X_{0}$ in 2.5. Let $M_{0}=M_{0}^{\prime} \cap M_{0}^{\prime \prime}$ and let $f_{0}, g_{0}$ be the restrictions of $\tilde{f}_{0}, \tilde{g}_{0}$ to $M_{0}$.

Definition 3.1. Let $\bar{N}_{f}(A, r)=N\left(\left[\operatorname{supp} f^{*} A\right], r\right)$ and define $\bar{N}_{g}(A, r)$ similarly.

LEMMA 3.2. $N_{f}(A, r)-N\left(R_{\hat{f}}, r\right) \leqslant \bar{N}_{f}(A, r)+O(1)$.

Proof. Using 2.8 and 2.7(c) we see that $\bar{N}_{f}(A, r)=\left(\left[\operatorname{supp} f^{*} A\right], \tau_{r} \alpha^{n-1}\right)$ and that $N_{f}(A, r)-N\left(R_{\hat{f}}, r\right)=\left(f^{*} A-R_{\hat{f}}, \tau_{r} \alpha^{n-1}\right)$. From $2.7(\mathrm{a})$ it follows that $\tau$ is plurisubharmonic on $B=\left\{x \in M: \tau(x)>\log r_{0}\right\}$. Thus $\alpha=(1 / 2 \pi) d d^{c} \tau$ is a positive semidefinite form on $B$. The complement of $B$ is compact. Since $\tau_{r}>0$, it is easy to see that if $D$ is a divisor on $M$ with $D \geqslant 0$, then there is a constant $c$ depending on $D$ such that $\left(D, \tau_{r} \alpha^{n-1}\right) \geqslant c$. To prove the lemma, it suffices to show that [supp $f^{*} A$ ] $-f^{*} A+R_{\hat{f}} \geqslant 0$ on $M$. In fact it suffices to show that if $p$ is any regular point of supp $f^{*} A$ lying in $M_{0}$, then there is a neighborhood $U$ of $p$ in $M_{0}$ such that $\left[\operatorname{supp} f_{0}^{*} A\right]-f_{0}^{*} A+\left.R_{\hat{f}_{0}}\right|_{U} \geqslant 0$ in $U$.

Assume $p$ is a regular point of $\operatorname{supp} f_{0}^{*} A$. Then there is a coordinate neighborhood $U_{1}$ of $p$ in $M_{0}$ with coordinates $\left(z_{1}, \ldots, z_{n}\right)$ such that $U_{1} \cap\left(\operatorname{supp} f_{0}^{*} A\right)=\{x$ $\left.\in U_{1}: z_{1}(x)=0\right\}$. Let $q=f(p) \in V$. There is a coordinate neighborhood $W$ of $q$ in $V$ with coordinates $\left(w_{1}, \ldots, w_{k}\right)$ such that for some $m<k, A \cap W=\{y \in W$ : $\left.w_{1} \cdots w_{m}(y)=0\right\}$. Let $U=U_{1} \cap f_{0}^{-1}(W)$. In local coordinates we can write $f_{0}$ : $U \rightarrow V$ as $f_{0}=\left(f_{1}, \ldots, f_{k}\right)$. Suppose $f_{i}(x)=z_{1}^{n_{i}} h_{i}(x)$ where $n_{i} \geqslant 0$ and $h_{i}$ is a holomorphic function on $U$ which does not vanish along supp $f_{0}^{*} A$. By computing the Jacobian matrix of $\hat{f}_{0}$ on $U$ in local coordinates and evaluating the determinant by expanding by minors along any row, it follows that the determinant has $\left(z_{1}(x)\right)^{\left(\Sigma_{i-1}^{k} n_{i}\right)-1}$ as a factor for some $n_{i}>0$. Hence

$$
\left.R_{\hat{f}_{0}}\right|_{U} \geqslant\left.\left(\left(\sum_{i=1}^{k} n_{i}\right)-1\right)\left[\operatorname{supp} f_{0}^{*} A\right]\right|_{U} .
$$

Now $\left.f_{0}^{*} A\right|_{U}=\left.\sum_{i=1}^{m} n_{i}\left[\operatorname{supp} f_{0}^{*} A\right]\right|_{U}$ giving $\left[\operatorname{supp} f_{0}^{*} A\right]-f_{0}^{*} A+\left.R_{\hat{f}_{0}}\right|_{U}>0$.

By Theorem 2.10,

$$
\| T_{f}\left(L_{A}, r\right)+T_{f}\left(K_{V}, r\right) \leqslant N_{f}(A, r)-N\left(R_{\hat{f}}, r\right)+o\left(T_{f}\left(L_{A}, r\right)\right)
$$

so by the previous lemma $\| T_{f}\left(L_{A}, r\right)+T_{f}\left(K_{V}, r\right) \leqslant \bar{N}_{f}(A, r)+o\left(T_{f}\left(L_{A}, r\right)\right)$. 
The same result holds if $f$ is replaced by $g$. Adding the two yields

$$
\begin{aligned}
\| T_{f}\left(L_{A}, r\right) & +T_{f}\left(K_{V}, r\right)+T_{g}\left(L_{A}, r\right)+T_{g}\left(K_{V}, r\right) \\
\leqslant & \bar{N}_{f}(A, r)+\bar{N}_{g}(A, r)+o\left(T_{f}\left(L_{A}, r\right)+T_{g}\left(L_{A}, r\right)\right) .
\end{aligned}
$$

By hypothesis 1.1(2), supp $f^{*} A=\operatorname{supp} g^{*} A$. Denote this common set by $E$; then $\bar{N}_{f}(A, r)=\bar{N}_{g}(A, r)=N(E, r)$. We next obtain a useful estimate for $N(E, r)$.

LeMma 3.4. $N(E, r) \leqslant T_{f}\left(i^{*} H, r\right)+T_{g}\left(i^{*} H, r\right)+O(1)$.

Proof. $V \times V$ is a smooth $2 k$-dimensional projective algebraic variety.

Define $h: M \rightarrow V \times V$ by $h=f \times g$ and $k: V \times V \rightarrow \mathbf{P}^{N} \times \mathbf{P}^{N}$ by $k=i \times i$. The function $h$ is meromorphic. Let $\pi_{i}: \mathbf{P}^{N} \times \mathbf{P}^{N} \rightarrow \mathbf{P}^{N}(i=1,2)$ be the projection onto the $i$ th factor. If $H$ denotes the hyperplane bundle on $\mathbf{P}^{N}$, let $\tilde{H}$ be the holomorphic line bundle on $\mathbf{P}^{N} \times \mathbf{P}^{N}$ defined by $\tilde{H}=\pi_{1}^{*} H \otimes \pi_{2}^{*} H$. Let $p_{i}: V \rightarrow V$ $\times V$ be projection on the $i$ th factor. Then $k^{*} \tilde{H}=\left(i \circ p_{1}\right)^{*} H \otimes\left(i \circ p_{2}\right)^{*} H$.

Let $\Delta$ be the diagonal on $\mathbf{P}^{N} \times \mathbf{P}^{N}$. Then there is a holomorphic section $\delta$ of $\tilde{H}$ such that

$$
\Delta \subset \operatorname{supp}(\operatorname{Div} \delta) \text { and } k \circ h(M) \nsubseteq \operatorname{supp}(\operatorname{Div} \delta) .
$$

To see this, let $\left(\left[z_{0}, \ldots, z_{N}\right],\left[w_{0}, \ldots, w_{N}\right]\right)$ be homogeneous coordinates on $\mathbf{P}^{N} \times \mathbf{P}^{N}$. Let $\left\{a_{k l}: 0 \leqslant k<l \leqslant N\right\}$ be an as yet undetermined set of complex constants not all of which are zero. Consider the bihomogeneous polynomial

$$
P\left(\left[z_{0}, \ldots, z_{N}\right],\left[w_{0}, \ldots, w_{N}\right]\right)=\sum_{0<k<l<N} a_{k l}\left(z_{k} w_{l}-z_{l} w_{k}\right) .
$$

$P$ determines a section $\delta$ of $\tilde{H}$ in a natural way having the same divisor as $P$. Clearly $\Delta \subset \operatorname{supp}(\operatorname{Div} \delta)$. Suppose for all choices of $\left\{a_{k l}\right\}, k \circ h(M) \subset$ $\operatorname{supp}\left(\operatorname{Div} \delta\right.$ ). Locally we can write $i=\left[i_{0}, \ldots, i_{N}\right]$. Then $P \circ k \circ h \equiv 0$ so that

$$
\sum_{0<k<l<N} a_{k l}\left[\left(i_{k} \circ f\right)\left(i_{l} \circ g\right)-\left(i_{l} \circ f\right)\left(i_{k} \circ g\right)\right] \equiv 0 .
$$

It follows that for all $0 \leqslant k<l \leqslant N$,

$$
\left(i_{k} \circ f\right)\left(i_{l} \circ g\right)-\left(i_{l} \circ f\right)\left(i_{k} \circ g\right) \equiv 0 .
$$

If $i$ is constant, Theorem 1.1 is trivial; so assume $i$ is not constant. Then locally, some $i_{k}$ is nonconstant, say $i_{0}$. Now $f_{0}$ and $g_{0}$ are nondegenerate so there exists an open set $U \subset M_{0}$ for which $f_{0}(U)$ and $g_{0}(U)$ are open. Since $i_{0}$ is nonconstant, it is nonconstant on each of the latter two open sets. Then $i_{0} \circ f_{0}$ and $i_{0} \circ g_{0}$ are nonconstant. In particular both $i_{0} \circ f_{0}$ and $i_{0} \circ g_{0}$ are nonzero on an open dense subset $M_{1}$ of $M_{0}$. From (3.7) it follows that

$$
\frac{i_{l} \circ f_{0}}{i_{0} \circ f_{0}} \equiv \frac{i_{l} \circ g_{0}}{i_{0} \circ g_{0}} \quad \text { for all } 1 \leqslant l \leqslant N \text { on } M_{1},
$$

yielding $i \circ f \equiv i \circ g$, which violates our assumption. So for some choice of $\left\{a_{k l}\right\}$, the $\delta$ associated to $P$ in (3.6) satisfies (3.5).

Since $E=\operatorname{supp} f^{*} A$, on $E_{0}=E \cap M_{0}, f_{0}=g_{0}$, and $(k \circ h)\left(E_{0}\right) \subset \Delta$ $\subset \operatorname{supp} \operatorname{Div} \delta$ giving

$$
[E] \leqslant \operatorname{Div}(\delta \circ k \circ h)=h^{*} \operatorname{Div}(\delta \circ k) .
$$


Now $\delta \circ k$ is a holomorphic section of $k^{*} \tilde{H}$. Furthermore, since $k \circ h(M) \nsubseteq$ $\operatorname{Div} \delta, h(M) \not \operatorname{Div} \delta \circ k$.

Applying Theorem 2.9 and 3.9, $N(E, r) \leqslant N_{h}(\operatorname{Div} \delta \circ k, r) \leqslant T_{h}\left(k^{*} \tilde{H}, r\right)+$ $O(1)$. Using 2.13,

$$
\begin{aligned}
T_{h}\left(k^{*} \tilde{H}, r\right) & =T_{f \times g}\left(\left(i \circ p_{1}\right)^{*} H \otimes\left(i \circ p_{2}\right)^{*} H, r\right) \\
& =T_{f}\left(i^{*} H, r\right)+T_{g}\left(i^{*} H, r\right)+O(1) .
\end{aligned}
$$

These last two inequalities give 3.4 .

Continuing the proof of 1.1 , using 3.4 in 3.3 gives

$$
\begin{aligned}
\| T_{f}\left(L_{A}, r\right)+ & T_{g}\left(L_{A}, r\right)+T_{f}\left(K_{V}, r\right)+T_{g}\left(K_{V}, r\right) \\
& \leqslant 2 T_{f}\left(i^{*} H, r\right)+2 T_{g}\left(i^{*} H, r\right)+o\left(T_{f}\left(L_{A}, r\right)+T_{g}\left(L_{A}, r\right)\right) .
\end{aligned}
$$

Setting $L_{1}=L_{A} \otimes K_{V} \otimes\left(i^{*} H\right)^{-2}$, we see that

$$
\| T_{f}\left(L_{1}, r\right)+T_{g}\left(L_{1}, r\right) \leqslant o\left(T_{f}\left(L_{A}, r\right)+T_{g}\left(L_{A}, r\right)\right) .
$$

By assumption, $L_{1}$ and $L_{A}$ are positive. Using the compactness of $V$ it is clear that there is a $b>0$ such that $c_{1}\left(L_{1}\right)-b c_{1}\left(L_{A}\right)>0$, hence such that $T_{f}\left(L_{1}, r\right)+$ $T_{g}\left(L_{1}, r\right)>b\left(T_{f}\left(L_{A}, r\right)+T_{g}\left(L_{A}, r\right)\right)$. By 3.10, b( $\left.T_{f}\left(L_{A}, r\right)+T_{g}\left(L_{A}, r\right)\right) \leqslant$ $o\left(T_{f}\left(L_{A}, r\right)+T_{g}\left(L_{A}, r\right)\right)$. Dividing by $T_{f}\left(L_{A}, r\right)+T_{g}\left(L_{A}, r\right)$ and letting $r$ approach infinity, we obtain $b \leqslant 0$, a contradiction. Thus $i \circ f \equiv i \circ g$.

\section{Applications of Theorem 1.1.}

THEOREM 4.1. Let $M$ be an $n$-dimensional smooth affine algebraic variety. Let $f, g$ : $M \rightarrow \mathbf{P}^{k}(\mathbf{C})$ be nondegenerate meromorphic maps, where $n \geqslant k$. Let $A$ be a hypersurface of degree at least $k+4$ in $\mathbf{P}^{k}$ having normal crossings. Suppose $f^{-1}(A)=$ $g^{-1}(A)$ as point sets and $f$ and $g$ agree at all points of $f^{-1}(A)$ lying in their common domain of determinacy. Suppose either $M=\mathbf{C}^{n}$ or $f$ and $g$ are transcendental. Then $f \equiv g$.

Proof. Let $H$ be the hyperplane bundle on $\mathrm{P}^{k}$. Let $d$ be the degree of $A$. Then the line bundle defined by $A$ is $L_{A}=H^{d}$. In Theorem 1.1 let $\mathbf{P}^{N}=\mathbf{P}^{k}$ and $i$ : $\mathbf{P}^{k} \rightarrow \mathbf{P}^{k}$ be the identity. Then $i^{*} H=H$. Taking $V=\mathbf{P}^{k}$ in 1.1 gives $K_{V}=$ $H^{-(k+1)}$.

We have $c_{1}\left(L_{A} \otimes K_{V} \otimes\left(i^{*} H\right)^{-2}\right)=c_{1}\left(H^{d-k-3}\right)=d-k-3>0$ if $d \geqslant k+$ 4. Thus $i \circ f \equiv i \circ g$ by 1.1 , so $f \equiv g$.

We have been unable to demonstrate the sharpness of 4.1 for the case $k=n$ and $n>1$. For $n=k=1$ and $M=\mathrm{C}$, the case which is Nevanlinna's original theorem, Nevanlinna gave an example to show that in order to conclude that $f \equiv g$, it is necessary to require $d \geqslant k+4=5$ : If $A=\{0, \infty, 1,-1\}$ and $f(z)=e^{z}, g(z)=$ $e^{-z}$, then all hypotheses of the theorem are met with the exception of the one concerning $d$, yet $f \neq g$; thus $d$ cannot be replaced by $d=k+3=4$. This example extends in an obvious manner to demonstrate the sharpness of 4.1 in the case $k=1, n$ is arbitrary, and $M=\mathbf{C}^{n}$.

The next theorem was first proved in the case $n=1$ and $M$ is an open Riemann surface having an infinite harmonic exhaustion and finite Euler characteristic by $E$. M. Schmid [14]. 
THEOREM 4.2. Let $M$ be an $n$-dimensional smooth affine variety and $V$ a smooth elliptic curve. Let $f, g: M \rightarrow V$ be nondegenerate meromorphic maps. Suppose there are five points $a_{1}, \ldots, a_{5}$ in $V$ for which $f^{-1}\left(a_{i}\right)=g^{-1}\left(a_{i}\right)$ as point sets, $i=$ $1, \ldots, 5$. If either $M=\mathbf{C}^{n}$ or $f$ and $g$ are transcendental, then $f \equiv g$.

Proof. We make the usual identification of $H^{2}(V, \mathbf{Z})$ with $\mathbf{Z}$. Let $L_{a_{i}}$ be the line bundle on $V$ determined by $a_{i}$. Let $A=\left\{a_{1}, \ldots, a_{5}\right\}$. If $L_{A}$ is the line bundle on $V$ determined by $A$, then $c_{1}\left(L_{A}\right)=5$. Since the tangent bundle of an elliptic curve is trivial, $c_{1}\left(K_{V}\right)=0$. Now let $i: V \rightarrow \mathbf{P}^{1}(\mathbf{C})$ be $i(z)=\wp(z)$ where $\wp$ is a Weierstrass $\wp$-function for $V . \wp$ exhibits $V$ as a two-sheeted cover of $\mathbf{P}^{1}$ branched at four points $e_{1}, e_{2}, e_{3}, e_{4}$. Let $H$ be the hyperplane bundle on $\mathbf{P}^{1} . c_{1}\left(i^{*} H\right)$ will be the number of zeros (counting multiplicities) of any holomorphic section of $i^{*} H$ on $V$. If $\left[w_{0}, w_{1}\right]$ are homogeneous coordinates on $\mathbf{P}_{1}$, there is a holomorphic section $\sigma$ of $H$ with Div $\sigma=\left\{\left[w_{0}, w_{1}\right] \in \mathbf{P}^{1}: w_{0}=0\right\}$. Then $\sigma \circ \wp$ is a holomorphic section of $i^{*} H$. Since $\wp$ is a two-to-one map (counting multiplicities) it follows that $\sigma \circ \wp$ has two zeros (counting multiplicities). Thus $c_{1}\left(i^{*} H\right)=2$. Now

$$
c_{1}\left(L_{A} \otimes K_{V} \otimes\left(i^{*} H\right)^{-2}\right)=c_{1}\left(L_{A}\right)+c_{1}\left(K_{V}\right)-2 c_{1}\left(i^{*} H\right)=1>0 .
$$

Also, $f^{-1}\left(a_{i}\right)=g^{-1}\left(a_{i}\right), i=1, \ldots, 5$, implies $f^{-1}(A)=g^{-1}(A)$ and $f \mid f^{-1}(A)=$ $g \mid f^{-1}(A)$. So by Theorem 1.1, $i \circ f \equiv i \circ g$, that is, $\wp \circ f \equiv \wp \circ g$.

The image of $f$ can omit no $a_{i}$. For if it does, then $N_{f}\left(a_{i}, r\right) \equiv 0$. By Shiffman's defect relation 2.12 ,

$$
\liminf _{r \rightarrow \infty}\left[1-\frac{N_{f}\left(a_{i}, r\right)}{T_{f}\left(L_{a_{i}}, r\right)}\right] \leqslant\left[c_{1}\left(K_{V}^{*}\right) / c_{1}\left(L_{a_{i}}\right)\right]
$$

giving $1 \leqslant 0$, a contradiction. Since there are five $a_{i}$ 's, there is some $a_{i}$, say $a_{1}$, which is not in $\left\{e_{1}, \ldots, e_{4}\right\}$, the branch locus of $\wp$. Then $\wp$ is one-to-one near $a_{1}$. Since $f^{-1}\left(a_{1}\right) \neq \varnothing$ and $g^{-1}\left(a_{1}\right)=f^{-1}\left(a_{1}\right)$, there is a $q \in f^{-1}\left(a_{1}\right) \cap g^{-1}\left(a_{1}\right)$. For all $z$ near $q, \wp \circ f(z)=\wp \circ g(z)$. Since $P$ is one-to-one near $f(q)=g(q), f(z)=g(z)$ for all $z$ near $q$. Thus $f \equiv g$ by uniqueness of analytic continuation.

For the case $n=1, M=\mathrm{C}$, Schmid [14] has already given an example showing the number five in 4.2 is sharp. The existence of a one-variable example demonstrating sharpness immediately implies the existence of an example demonstrating sharpness for $n>1$.

It is interesting to note that Theorem 4.2 shows that sharp results cannot always be obtained from Theorem 1.1 if $i$ is taken to be an embedding. If one were to try to obtain 4.2 by finding an embedding $i: V \rightarrow \mathbf{P}^{N}(\mathbf{C})$ and concluding that $i \circ f \equiv$ $i \circ g$, hence $f \equiv g$, by showing that $c_{1}\left(L_{A} \otimes K_{V} \otimes\left(i^{*} H\right)^{-2}\right)>0$ then he would need $c_{1}\left(i^{*} H\right)=0,1$, or 2 . We will show this condition cannot be met by applying the following corollary of the Riemann-Roch Theorem from [11]:

Proposition 4.3. Let $E$ be a holomorphic line bundle on a compact Riemann surface $X$ of genus $g$. If $c_{1}(E) \geqslant 2 g-2$ then $\operatorname{dim} H^{0}(X, O(E))=c_{1}(E)-(g-1)$, unless $E=K_{X}$, in which case $\operatorname{dim} H^{0}(X, \mathcal{O}(E))=g$. 
From this proposition it follows that on $V$, if $c_{1}\left(i^{*} H\right)=0,1$, or 2 then $\operatorname{dim} H^{0}\left(V, \vartheta\left(i^{*} H\right)\right)=0$, 1 , or 2 . Thus $i(V)$ would be contained in a one-dimensional subspace of $\mathbf{P}^{N}(\mathbf{C})$, which is impossible if $i$ is an embedding.

5. Further remarks. Theorem 1.1 is false if the hypothesis that $f(z)=g(z)$ for all $z$ in $f^{-1}(A)$ which lie in the common domain of determinacy of $f$ and $g$ is dropped. The problem of determining whether, in the absence of this hypothesis, there must nevertheless exist some sort of nice relation between $f$ and $g$ has not yet been solved. Two pertinent examples follow.

EXAMPLE 5.1. Let $f: \mathbf{C}^{n} \rightarrow \mathbf{P}^{n}(\mathbf{C})$ be any nondegenerate meromorphic map given by $\left[f_{0}, \ldots, f_{n}\right]$ where each $f_{i}: \mathbf{C}^{n} \rightarrow \mathbf{C}$ is holomorphic. Consider the smooth hypersurface $A$ of degree $d \geqslant n+4$ in $\mathbf{P}^{n}$ defined by the homogeneous equation $z_{0}^{d}+\ldots+z_{n}^{d}=0$. It is easy to see from the defect relation 2.12 that $f^{-1}(A) \neq \varnothing$. Some $f_{i}$, say $f_{0}$, is not identically zero on $f^{-1}(A)$. Let $\omega$ be any $d$ th root of unity. Define $g: \mathbf{C}^{n} \rightarrow \mathbf{P}^{n}$ by $g=\left[\omega f_{0}, f_{1}, \ldots, f_{n}\right]$. Then $g^{-1}(A)=f^{-1}(A)$, but $g \mid f^{-1}(A)$ $\neq f \mid f^{-1}(A)$ and $f \neq g$. Thus the hypothesis cannot be dropped in Theorem 4.1.

Example 5.2. Let $f, g: \mathbf{C} \rightarrow \mathbf{P}^{1}(\mathbf{C})$ be $f(z)=\sin z, g(z)=\cos z$. Let $A=\{1 / 2$, $-1 / 2, \sqrt{3} / 2,-\sqrt{3} / 2, \infty\}$. Then $f^{-1}(A)=g^{-1}(A)$, but $f\left|f^{-1}(A) \neq g\right| f^{-1}(A)$ and $f \neq g$. Once again the hypothesis cannot be dropped in Theorem 4.1.

Theorem 1.1 is also false if the hypothesis that $A$ has normal crossings is dropped.

EXAMPLE 5.3. Let $\mathbf{P}^{2}(\mathbf{C})$ have homogeneous coordinates $\left[w_{0}, w_{1}, w_{2}\right]$. Let $k \geqslant 6$. Let $H_{1}, \ldots, H_{k}$ be $k$ distinct hyperplanes in $\mathbf{P}^{2}$ defined by linear homogeneous equations of the form $L_{i}\left(w_{1}, w_{2}\right)=0, i=1, \ldots, k$. Define $f: \mathbf{C}^{2} \rightarrow \mathbf{P}^{2}$ by $f\left(z_{1}, z_{2}\right)$ $=\left[1, z_{1}, z_{2}\right]$. Define $g: \mathbf{C}^{2} \rightarrow \mathbf{P}^{2}$ by $g\left(z_{1}, z_{2}\right)=\left[1, z_{1} \varphi\left(z_{1}, z_{2}\right), z_{2} \varphi\left(z_{1}, z_{2}\right)\right]$ where $\varphi\left(z_{1}, z_{2}\right)=\exp \left(\prod_{i=1}^{n} L_{i}\left(z_{1}, z_{2}\right)\right)$. Then $L_{i}\left(f\left(z_{1}, z_{2}\right)\right)=0$ if and only if $L_{i}\left(g\left(z_{1}, z_{2}\right)\right)=$ 0 . Furthermore, if $L_{i}\left(f\left(z_{1}, z_{2}\right)\right)=0$ then $g\left(z_{1}, z_{2}\right)=f\left(z_{1}, z_{2}\right)$. Let $A=H_{1}$ $\cup \ldots \cup H_{k} . A$ is a hypersurface of degree $k \geqslant 6$ in $\mathbf{P}^{2}$ without normal crossings. $f$ and $g$ are nondegenerate, $f^{-1}(A)=g^{-1}(A), f\left|f^{-1}(A)=g\right| f^{-1}(A)$ but $f \neq g$. Thus, the normal crossings hypothesis cannot be removed in Theorem 4.1.

As stated, hypothesis (1) of Theorem 1.1 requires that when $M \neq \mathbf{C}^{n}$, both $f$ and $g$ should be transcendental. This hypothesis may be weakened, with no change in the rest of the theorem's statement, to require only that at least one of $f$ and $g$ is transcendental. For, suppose $f$ is transcendental and $g$ is rational. As is well known, $\log r=o\left(T_{f}\left(L_{A}, r\right)\right)$ and $T_{g}\left(L_{A}, r\right)=O(\log r)$. Theorem 2.10 still holds for $f$. For $g$, $[15,3.2]$ enables us to conclude that

$$
\| T_{g}\left(L_{A}, r\right)-\bar{N}_{g}(A, r)+T_{g}\left(K_{V}, r\right) \leqslant N\left(R_{\pi}, r\right)+o\left(T_{f}\left(L_{A}, r\right)\right) .
$$

Now $N\left(R_{\pi}, r\right)=O(\log r)=o\left(T_{f}\left(L_{A}, r\right)\right)$.

The reasoning of $\$ 3$ shows 3.3 is still valid, and the remainder of the proof of 1.1 is the same. Theorems 4.1 and 4.2 may be similarly weakened.

The following example, based on an idea of Paul Roberts, shows that in the case $\boldsymbol{M} \neq \mathbf{C}^{n}$ the hypothesis that $f$ or $g$ is transcendental may be essential.

EXAMPLE 5.4. Let $V$ be an elliptic curve which is a nonsingular cubic in $\mathbf{P}^{2}(\mathbf{C})$. Give $V$ an additive group structure in the standard manner, and let 0 be the 
identity. Let $b$ be a point in $V$ of order five, and let $c, d$ be two distinct points of order two in $V$. Since $0+2 b+3 b=0$, there is a line in $\mathbf{P}^{2}$ intersecting $V$ in $\{0,2 b, 3 b\}$. Thus $M=V \sim\{0,2 b, 3 b\}$ is affine. Define $f, g: M \rightarrow V$ by $f(z)=z$, $g(z)=-z$. If $z=0,2 b$, or $3 b$ then $f^{-1}(z)=g^{-1}(z)=\varnothing$. If $z=c, f^{-1}(z)=g^{-1}(z)$ $=c$. If $z=d, f^{-1}(z)=g^{-1}(z)=d$. Thus there are five points $a_{i} \in V$ such that $f^{-1}\left(a_{i}\right)=g^{-1}\left(a_{i}\right)$. Clearly $f \not z g$; but here $M \neq \mathbf{C}^{n}$ and both $f$ and $g$ are rational, so that Theorem 4.2, improved as indicated above, does not apply.

Whether variants of Theorem 1.1 can be obtained for the case that $A$ has singularities other than those of the normal crossing type has yet to be determined.

It should be mentioned that since the method of proof of 1.1 uses Shiffman's First and Second Main Theorems in an essentially computational way, one should be able to use other types of "Main Theorems" in the same way to obtain similar results in different settings.

\section{BIBLIOGRAPHY}

1. J. A. Carlson, Personal correspondence.

2. H. Cartan, Sur quelques théorèmes de M. R. Nevanlinna, C. R. Acad. Sci. Paris 185 (1927), 1253-1254.

3. Un nouveau théorème d'unicité relatif aux fonctions méromorphes, C. R. Acad. Sci. Paris 188 (1929), 301-303.

4. Sur les zéros des combinaisons linéaires de p fonctions holomorphes données, Mathematica (Cluj) 7 (1933), 5-31.

5. S. J. Drouilhet, $A$ unicity theorem for equidimensional holomorphic maps, Proc. Sympos. Pure Math., vol. 30, Part 2, Amer. Math. Soc., Providence, R. I., 1977, pp. 237-238.

6. H. Fujimoto, The uniqueness problem of meromorphic maps into the complex projective space, Nagoya Math. J. 64 (1975), 1-23.

7. A uniqueness theorem of algebraically non-degenerate meromorphic maps into $P^{N}(C)$, Nagoya Math. J. 64 (1976), 117-147.

8. Remarks to the uniqueness of meromorphic maps into $P^{N}(C)$. I, II, Nagoya Math. J. 71 (1978), 13-41.

9. , Remarks to the uniqueness of meromorphic maps into $P^{N}(C)$. III, Nagoya Math. J. 75 (1979), 71.

10. P. A. Griffiths and J. King, Nevanlinna theory and holomorphic mappings between algebraic varieties, Acta Math. 130 (1973), 145-220.

11. R. C. Gunning, Lectures on Riemann surfaces, Princeton Univ. Press, Princeton, N. J., 1966.

12. R. Nevanlinna, Einige Eindeutigkeitsätze in der Theorie der meromorphen Funktionen, Acta Math. 48 (1926), 367-391.

13. L_ Le théorème de Picard-Borel et la théorie des fonctions méromorphes, Gauthier-Villars, Paris, 1929.

14. E. M. Schmid, Some theorems on value distributions of meromorphic functions, Math. Z. 120 (1971), 61-92.

15. B. Shiffman, Nevanlinna defect relations for singular divisors, Invent. Math. 31 (1975), 155-182.

Department of Mathematics, Yankton College, Yankton, South Dakota 57078 